\title{
On Skopos Theory: An Exemplification of Fiction
}

\author{
Cai Ning \\ College of Electrical Engineering \\ Northwest University for Nationalities \\ Lanzhou, China \\ E-mail: caining91@tsinghua.org.cn
}

\author{
Zhou Jian* \\ College of Electrical Engineering \\ Northwest University for Nationalities \\ Lanzhou, China \\ E-mail: 397125172@qq.com \\ *Corresponding Author
}

\begin{abstract}
The Skopos Theory is one of the most recognized theories in modern translation field. The 'Skopos Theory' is originated in Germany, and then it becomes widely accepted and recognized in modern translation study field. The meaning of Skopos in Latin means aim, purpose, intention or function. The key idea in Skopos Theory is that the translation action is the way to deliver information, which means, the translations don't need to be word by word but need to present the key meaning from source text. It is found that, translators always cannot deliver all the meaning by translating the source text word by word. It adds the subjective initiative based on equivalent translation Theory, which becomes more real and easily accepted by people. The Chinese edition of Harry Potter and the Philosopher's Stone from People's Publishing House in $\mathbf{2 0 0 0}$ is widely accepted by the Chinese readers. Based on this research model, this paper expects to analyze the reasons behind the success of Harry Potter and the Philosopher's Stone and its translation from the perspective of 'Skopos Theory' and improve the practical skills in translation field. The current paper analyzes the author, the story and the Chinese edition of answer: why is it so successful in China? It compares the Chinese version and English version of Harry Potter and the Philosopher's Stone. It includes the translation of proper nouns, the translation of Chinese idioms, and the translation of sentences.
\end{abstract}

Keywords- Translation; Target Audience; Skopos Theory; Quality; Idiom

\section{INTRODUCTION}

The worldwide success of Harry Potter and the Philosopher's Stone is due to not only the reasonable arrangements of the story clue and the appropriate expressions of the main characters, but also the success in its translation. The 'Skopos Theory' is one of the most recognized theories in modern translation field. It adds the subjective initiative based on equivalent translation theory, which becomes more real and easily accepted by people. The Chinese edition of Harry Potter and the Philosopher's Stone from People's Publishing House in 2000 is widely accepted by the Chinese readers. Based on this research model, this paper expects to analyze the reasons behind the success of Harry Potter and the Philosopher's Stone and its translation from the perspective of 'Skopos Theory' and improve the practical skills in translation field.

Many researchers have conducted studies in this topic. From the aspect of children's literature, it is found that in order to make this translation suitable to children readers' demands, the translators didn't strictly comply with [1-3] 'loyalty principle' under the 'Skopos Theory'. Instead, plenty of vivid descriptions are used to present imaginary sceneries, which creates a strong sense of pictures in readers' mind [1-3]. However, some studies have found that because of 'Skopos Theory' on translation, the Chinese version is constrained to the original copies, and those translations are not satisfactory. Those weaknesses lead to the loss of essence in original copies [4]. The translations of some parts in Chinese version are regarded as "too long and misunderstanding" [5]. All the previous studies comment on the translations from several aspects, but few comment from the Skopos Theory. Therefore, in this paper, the analysis of translation from the aspect of Skopos Theory will be presented, which has discovered a different view from previous studies.

The book was published on 26 June 1997 and soon became a boom in modern novel field. In 1998, Harry Potter and the Philosopher's Stone won most of the British book rewards that were judged by children, and many other rewards in the USA. In the coming year, the book reached the top of the New York Times list of best-selling fiction, and stayed in the top of the list for the following two years. From the success of the series, it is safe to conclude that this is a book worth researching. "Skopos Theory" is one of the most popular translation theories in modern days. Based on the reasons mentioned above, this study is conducted out of the following purposes.

First of all, by analyzing the success of the Harry Potter and the Philosopher's Stone the story itself, it can observe the influence of translation. Secondly, 'Skopo Theory' is a new perspective to evaluate the translation.

The current paper will analyze the author, the story and the Chinese edition to answer: why is it so successful in China? It will compare the Chinese version and English version of Harry Potter and the Philosopher's Stone. It includes the translation of proper nouns, the translation of Chinese idioms, and the translation of sentences.

\section{AN OVER VIEW OF SKOPOS THEORY AND ITS APPLICATION IN TRANSLATION}

The 'Skopos Theory' is originated in Germany, and then it becomes widely accepted and recognized in modern translation study field. The meaning of Skopos in Latin means aim, purpose, intention or function. The key idea in Skopos Theory is that the translation action is the way to deliver information, which means, the translations don't need to be word by word but need to present the key meaning from source text. 
It is found that, translators always cannot deliver all the meaning by translating the source text word by word. When the translators highlight some of the meanings, the other parts of the source text always are suppressed. Then, it is not surprising that the translation activities always sacrifice parts of the original text [6]. Thus, for translators, it is important to decide which parts to keep and which parts to omit. The Skopos Theory points out the audience is the most important role, so the purpose of the source text has the highest priority. The audience is the readers of translated text. The characteristic of the audience is they have their own background knowledge, the expectation of the target text and the requirement of the meanings [7]. In Harry Potter and Philosopher's Stone, the audience is the readers in Chinese mainland, most of the readers are children.

The 'Skopos Theory' mainly has three principles, namely: Skopos Rule, Coherence Rule and Fidelity Principle, the Principle of Loyalty.

The first rule requires the translators to translate from the perspective of target language which puts the reader's reading experience in the first place. This rule has the highest priority in the whole theory. The process to achieve the purpose of translation determines the translation action itself, which means the results guide the method. There always are three purposes during the translation activities. The first one is the basic translation; the second one is the communicative purpose of the translation, such as inspire the readers; the last one is to use some special method to translation, such as to explain the special grammatical structure in a language. Mostly, the purpose is the communicative function. So the translator according to this to decide the translation methods: literal translation or free translation [8].

The second rule and the third rule is not generally suitable for every case, therefore, it is less important than the first rule. Coherence Rule means the target text must meet the intra textual coherence standard. In other words, the target text needs to require readability and acceptability [9]. Loyalty principle focuses on the relationship between the main concern in the process of translation, the translator and the author; the translator should follow the principle of "function plus loyalty" to improve the translation activities [10].

Another aspect of Skopos Theory is regarded as "Adequacy but not Equivalence". To be more specific, the target text needs to satisfy the target audience but it doesn't mean the translator needs to translate word by word. The target text and the source text have the difference sentence but express the same meaning. Equivalence is not a "must" criterion to judge the translation. It is only the static form of dynamic Adequacy. So it is important to consider the potential function of the original text [11].

This section explained what the Skopos Theory is and listed out important rules inside. The significant concept inside the theory is the priority of the target audience. In other words, the translators do not necessarily translate the source text word by word, but the more important part is the target text. Also the criteria of commenting a translation is not how it equals to the source text but how adequate and accurate it delivers the original meaning.

To conclude, 'Skopos Theory' is an important theory in translation, and Harry Potter and the Philosopher's
Stone is the most typical literature. So it is reasonable to take these two into research.

\section{REASONS FOR THE DIFFERENCES OF ANIMAL WORDS IN CHINESE AND WESTERN CULTURES}

\section{A. Overview}

In this section, it is designed to analyze the translation from the aspect of words and phrases to conclude the basic rules and tips in translation activities. The analyzing of words mainly includes the proper nouns the former is the special one in magic stories while the latter has more influence in Chinese readers. Thus, the analysis is guiding to the whole study; after the analyzing of the words, it moves on to the phrases. The phrases and sentences help readers understand the whole story.

When introduce the Harry Potter and philosopher's stone, Rowling admits that there is no specific age groups of readers', it is a novel for all age readers. It has a series of lovers from all ages, even in some countries also published two versions: one is translate for adults and the other version is for children, even though the content is exactly the same [11].

When Rowling introduce about her book, she said the book has lots of things about death, she said "My book is closely related to death. The start of the series is Harry's parents died, through which Voldemort is addicted to conquer death, and at all cost to achieve the goal. I was able to understand Voldemort's fear of death, because this fear was shared by all of us" [12].

Generally, for the idea of the series mainly are: normal, oppression, survival, overcome difficulties, youth learn with their fear of growth. The series also have loads of moral content, with justice and evil against the fierce and obvious, some people face an important choice, and the shadow of humanity was also demonstrated. Life, death, love, friendship and braveness are the important role in the series. So it can be conclude the audience for the book is more than for children. So when doing the translation, the translator needs to set target audience for all ages [13].

\section{B. Translation of Proper Nouns}

\section{1) Broomsticks/Brooms}

In the story, Broomsticks or Brooms are not ordinary brooms to clean the floor but magic tools, which are used by the wizard to ride and fly. They are transportation tools instead of cleaning tools. In the translation, translators add adjective to define the function of the broom. "Fei-Tian" means fly directly into sky, which seems to be magic and powerful, this consciously describes the special aspect of the brooms. "Sao-Zhou" simply means broomsticks/ brooms in Chinese. This combination and expansion of the word shows the properties of the Skopos Theory: Not limited in the word-by-word translation, but combine the target ideas with original meanings of the words.

\section{2) Dementors}

In the Harry Potter Series, Rowling described them as soulless creatures, which constantly absorb human minds of happiness and intelligence. The presence of Dementors makes the surrounding atmosphere become cold and dark. The translation of Dementors is "She-Hun-Guai". The Chinese character "She" has the meaning of absorbing or depriving. "Hun" simply means the soul of the creatures. 
"Guai" means some strange creatures like monster or cockatrice which is harmful to human-body from both physical and psychology aspects. The expression of this words aims at making readers feel scary about these creatures. This translation clearly delivered the feeling of scariness inside this creature, which makes readers have a better understanding of what Rowling is talking about. Even though the pronunciation does not stay the same, but the meanings are still well expressed.

3) Gringotto

The Gringotto, in the stories, is described as a snowwhite building. The author uses this word to refer to the bank of the wizard, which is an old building guarded by the goblins. It was translated according to its pronunciation as "Gu-Ling-Ge". Besides, the translation is corresponding to its meaning. "Gu" is a multi-meaning word, which has the meaning of old or strange. "Ling" as an adjective means smart and it also means goblin as a noun. "Ge" in Chinese culture means a building which is full of Chinese cultural elements. So the translation of "Gu-Ling-Ge" helps the Chinese readers to imagine an ancient antique magic building.

\section{4) The Leaky Cauldron}

In the Chapter Five in the book, Rowling described it as a "tiny, grubby-looking" pub, dark and shabby. The word Cauldron means a large pot that is used for boiling water, the direct meaning of leaky is the hole in the container which permits the unwanted access of fluid. These two words are together to mean the tear and unworthy bar. It is translated as "Po-Fu-Jiu-Ba". In the western culture, Cauldron refers to a special bar which is used to make boilers and to sell bears. While in Chinese, "Po" is used to describe as torn out and shabby, "Fu" has the meaning of boiler. These two characters consciously describe the tiny shabby bar. As a result, the translation of "Po-Fu-Jiu-Ba" shows the meaning the author wants to express in the context, and it's in accordance with the rules of Skopos Theory.

5) Lord Voldemort

In Harry Potter Series, Lord Voldemort was called "the Dark Lord" or "You-Know-Who", who is an enemy of Harry Potter and he is the one who wants to conquer the world. J. K. Rowling described him as a powerful evil wizard, who is too strong to be called by name directly from others. This character is translated as "Fu-Di-Mo" in Chinese which is considered to be proper. The character "Fu" in China means to surrender and to hide. "Mo" means some magic character with evil personality. By combining all these three characters together, "Fu-Di-Mo" becomes a brilliant translation. It is not only corresponding to its original pronunciation, but also in accordance with Chinese culture. The target of the translation here is to show the personality of Lord Voldemort: Powerful and Scary. By glancing at this noun, the audience can feel the evil power inside the three characters, especially the character of "Mo".

6) Muggle

In the Harry Potter Series, non-magical people are often described as foolish, who are completely ignorant of the Wizard world. The term Muggle is sometimes used in a bad manner in the stories. For instance, Draco Malfoy, who is born in a magic family, has discrimination in nonmagic people. So when he talks about non-magical people.
He always uses the word muggle to show his discrimination.

Muggle refers to a person who is a member of the nonmagical community; Muggles are simply ordinary human beings rather than witches and wizards. "Ma" in Chinese means not so clever or with a lifeless mind, which refers to those people who are not active or intelligent. "Gua" to some degree, stands for a group of people who are not as clever as others. This word is corresponding to the context in Harry Potter Series when people talk about Muggles. Also, the pronunciation of "Ma-Gua" is quite alike Muggle. So this translation delivers the meaning as well as the pronunciation which leads to be a good translation.

\section{Translation of Magic Phrases}

\section{1) Petrificus Totalus}

"Petrificus Totalus" is a magic phrases Hermione used in Chapter 16 (p.198) which refers to make everything not move. In Chinese, translators translate it as "Tong-TongShi-Hua", this short phrase means totally stay stable. The short term makes this magic phrase in rhythm, and not too wordy. This translation highly attached the importance of the feeling of audience, which is corresponding with the Skopos Theory.

\section{2) Expecto Patronum}

"Expecto Patronum" is another magic phrase translated as "Hu-Shen-Hu-Wei" which refers to call up Patrons to protect them. "Patrons" in Chinese means "Shou-HuShen" which can be simplified as "Shen". Chinese always consider the magic phrases as short sentences with rhythm. The translator took this idea into consideration; translate the "Expecto Patronum" as "Hu-Shen-Hu-Wei" which clearly shows the meaning and has rhythm inside. So it can be said this translation takes the target audience into high priority, which corresponds to the Skopos Theory.

\section{3) Crucio}

"Crucio" is one of dark magic imprecation which translates as "Zuan-Xin-Wan-Gu". In the source text, the writer describes it as very bad magic words which are rarely used. When the wizard use it, the people under the spell can feel full of pain, the pain will be a caster, if it does not stop, the pain may lead people go mad and even death. This is a really terrible phases, and it is translated as proper phrases "Zuan-Xin-Wan-Gu". By seeing the words, the readers can feel the pain of this spell. This translation shows the priority of readers and corresponds to the Skopos Rule, and the Principle of Loyalty in the Skopos Theory.

\section{4) Imperio}

"Imperio" is another dark magic spell which translates as "Hun-Po-Chu-Qiao". Different from other translations, this phrase is existed in the Chinese culture. "Hun-Po-ChuQiao" is explanted as "the soul is separate from the body". As in traditional Chinese meaning, when somebody's soul is separated from the body, the feeling of the breathless may happened. And it may have some strange things happened [14-16]. In the series, this is a terrible evil magic. It leads to the loss of conscious, and the intentions. It cannot be self-recovered, only by others saving the conscious can be recovered. The translator has a good use of the existed words; this makes more sense to help the audience understand the idea original text delivered. Uggle refers to a person who is a member of the non- 
magical community; Muggles are simply ordinary human beings rather than witches and wizards. "Ma" in Chinese means not so clever or with a lifeless mind, which refers to those people who are not active or intelligent. "Gua" to some degree, stands for a group of people who are not as clever as others. This word is corresponding to the context in Harry Potter Series when people talk about Muggles. Also, the pronunciation of "Ma-Gua" is quite like Muggle. So this translation delivers the meaning as well as the pronunciation which leads to be a good translation.

\section{CONCLUSIONS}

Based on the 'Skopos Theory', this paper analyzes the translation of Harry Potter and the Philosopher's Stone from People's Literature Publishing House in 2000

First part mainly concentrates on the theory. It includes Skopos rule, coherence rule and fidelity principle, the principle of loyalty. The first rule require the translators translate from the perspective of target language which puts the reader's reading experience in the first place. Because of the consideration of the coherence and unity and other factors in a clear expression of the original author's objective, this rule brings good translation experience. It is considerate that the most important rule, which refers that the principle of translation should be in the target language situation. Translation requirements is the key concept, translator needs to find what is needed to express the right meaning, and the translator is not passively accept everything. Coherence rule and fidelity principle means that it is important to meet the coherence criteria, which refers to the translation being able to let the readers understand the meaning. The principle of loyalty means there must be an inter-textual coherence between the source texts and target texts, which is similar to the fidelity to the source texts.

After knowing the basic knowledge of the theory, this paper reveals background of the research, which contributes to the understanding of the culture.

Finally, it comes to the main body part of this paper: the analysis of translation. The translation of the titles shows the difference in translating using the theory and without it. Then by comparing Chinese and English version of the Harry Potter series, it has found that the specialty of the translation lies in the translation of nouns, magic terms, and some phrases. All these analyses tend to show the good parts of the translations. However, there are some shortcomings in the translation. From the aspect of the cultural difference, the multiple meaning of the words and the translation is too complicated.

\section{ACKNOWLEDGMENT}

This work is supported by the Fundamental Research Funds for the Central Universities of China for Northwest University for Nationalities (Grant No. 31920150007), the Zhejiang Open Foundation of the Most Important Subjects Program, the Project for Young Talents of State Ethnic Affairs Commission (SEAC) of China (Grant [2013] 231), and by National Natural Science Foundation (NNSF) of China (Grants 61263002 \& 61374054).

The authors would like to express thanks to Prof. M. J. Khan with the School of PN Engineering, National University of Sciences \& Technology, Islamabad, Pakistan and Prof. J. Cao with the Research Institute of Information Technology, Tsinghua University, Beijing, China for the beneficial discussions about this interesting topic.

\section{REFERENCES}

[1] X. Wei, Children Literature Translation Under Perspective of Adaptive Theory, Shanxi Normal University, 2014.

[2] Y. Kang, Analysis of Mainland and Taiwan Versions of Harry Potter and Philosopher's Stone from Perspective of Subculture, Hebei Agricultural University, 2013.

[3] Y. Wang and Z. Yue, "Spell translation in Harry Potter from the perspective of Skopostheorie: A comparative analysis on the versions from Mainland China and Taiwan", Studies in Literature and Language, vol. 7, pp. 119-124, 2013.

[4] K. Reiss, and H. J. Vermeer, Towards a General Theory of Translational Action: Skopos Theory Explained, Routledge, 2014.

[5] J. Munday, Introducing Translation Studies: Theories and Applications, Routledge, 2012.

[6] Y. Zhao and Y. Jiang, "Translation techniques of Chinese union version: From the perspective of Skopos Theory", Canadian Social Science, vol. 10, pp. 9-13, 2014.

[7] L. Tack, "Review of translating as purposeful activity: functionalist approaches explained by Christiane Nord", Target, vol. 13, pp. 197-197, 2002.

[8] P. Flynn, "Skopos Theory: An ethnographic enquiry", Perspectives, vol. 12, pp. 270-285, 2004.

[9] N. Jiang, On English-Chinese Children Literature Translation via Teleology: A Case Study of Chinese Version of Harry Potter From Perspective of Skopostheorie, Suzhou University, 2010.

[10] S. Wang, Translation Under a Context of Mass Culture, Hebei Normal University, 2008.

[11] C. A. Ellwood, An Introdution to Social Psychology, Hardpress Publishing, 2013.

[12] E. Sapir, Language-An Introduction to the Study of Speech, New York: Harcourt, Brace and Company, 1921.

[13] E. Sapir, "Culture, Genuine and Spurious", Am. J. Soc., vol. 29, pp. 401-429, 1924.

[14] L. Xue, "The application of traditional language learning strategy", J. Univ. Sci Tech. Beijing (Social Sciences Edition), vol. 6, pp. 114-116, 2008.

[15] L. Xue, "The comparison between traditional Chinese literacy strategies and English vocabulary strategies", Sino-US English Teaching, vol. 1, pp. 42-45, 2009.

[16] C. A. Ellwood, An Introdution to Social Psychology, Hardpress Publishing, 2013. 